\title{
The Preparation and Analytical Study of Conducting Polyaniline Thin Films
}

\author{
Saad Lamouri* ${ }^{*}$, Soumia Bendahgane ${ }^{1}$ and Atika Oudia ${ }^{2}$ \\ ${ }^{1}$ Laboratoire de Chimie Macromoléculaire, Ecole Militaire Polytechnique, BP 17, Bordj El Bahri, 16111, Algeria \\ ${ }^{2}$ Faculty of Science, Departement of Chimistry, Universidade da Beira Interior, 6201-001 Covilhã, Portugal
}

\begin{abstract}
Polyaniline free-standing films behavior has been investigated by electrical measurements, spectral, thermal and morphological analyzes. Two categories of thin films were elaborated. The first one represents films containing intrinsic conducting polymer, Polyaniline (PANI), doped by dipping method in sulfanilic acid solutions for various period of time. The second category contains conducting polymer composite; Polyaniline-Polyvinylchloride (PANI/ PVC), prepared by co-dissolution method using sulfanilic acid as a doping agent and N-methylpyrrolidone (NMP) as solvent and deposited on glass substrate using the spin coating technique. The effect of doping time, PANI fraction and film thickness on the conductivity has been studied principally by electrical measurements.
\end{abstract}

Keywords: Thin films; PANI; Sulfanilic acid; Composite PANI/PVC; Spin coating

\section{Introduction}

Electronic conducting polymers in general have been studied for more than 20 years [1]. Among conducting polymers, polyaniline is regarded as one of the most technologically promising electrically conductive polymers due to its simple synthesis, good environmental stability and adequate level of electrical conductivity [2]. Like any intrinsic conducting polymer, the PANI is a material difficult to transform that cannot be handled like the traditional thermoplastic polymers [3]. This characteristic is related to the bond alternation in its long chain. Due to its molecular characteristics, PANI is also infusible and practically insoluble in common organic solvents [4]. In order to overcome this drawback, many research groups enhanced the solubility of conjugated polymers by using functionalized dopants $[3,5,6]$. Another strategy is based on the preparation of blends and composites with insulator polymers to obtain combinations of desired properties that cannot be obtained from the individual components [3]. At the present time, a big part of the research efforts in the conducting polymers field is dedicated to the developments and the optimization of their implementation either as an intrinsic conducting polymer, or by the formation of a composite with good mechanical properties [7]. Main criteria required for most applications is not only a metal-like electrical property, but the combination of electrical conductivity and polymeric properties such as flexibility, low density, and ease of structural modification that suffice for many commercial applications, especially in the fields of the electrostatic loads dissipation and electromagnetic interferences [8]. In the present research, sulfanilic acid has been used as a new functionalized dopant to elaborate thin films of polyaniline either as an intrinsic conducting polymer, or by the preparation of a composite PANI/PVC. The PVC has been chosen here as an insulating matrix because of its solubility in the N-methylpyrrolidone (NMP) which has been used as solvent of PANI.

\section{Materials and Methods}

\section{Synthesis of polyaniline emeraldine base $\left(\mathrm{PANI}_{\mathrm{EB}}\right)$}

The polyaniline was synthesized by chemical oxidation of aniline

\begin{tabular}{|c|l|l|l|l|l|l|l|}
\hline PANI concentration in NMP $(\mathbf{w} \%)$ & 1.0 & 1.5 & 2.0 & 2.5 & 3.0 & 3.5 & 4.0 \\
\hline Thickness $(\boldsymbol{\mu m})$ & 0.6 & 1.0 & 2.0 & 2.6 & 3.3 & 5.3 & 6.0 \\
\hline
\end{tabular}

Table 1: Concentration of PANI solution and PANI thickness. with the ammonium persulphate $\left\{\left(\mathrm{NH}_{4}\right)_{2} \mathrm{~S}_{2} \mathrm{O}_{8}\right\}$ in an aqueous $\mathrm{HCl}$ medium prepared into a ratio of concentration [HCl]/[aniline] equal to 6 . The reaction was carried out at low temperature $\left(-5^{\circ} \mathrm{C}\right)$. The obtained polyaniline hydrochloride was converted to the base form by stirring with $0,1 \mathrm{M}$ aqueous $\mathrm{NH}_{3}$ solution for 72 hours. The PANI was filtered by vacuum filtration and washed several times with distilled water and ethanol, and then the powder was dried in vacuum for $48 \mathrm{~h}$ at $60^{\circ} \mathrm{C}$.

\section{Preparation of polyaniline films}

The PANI ${ }_{\mathrm{EB}}$ films were prepared by casting PANI solution in NMP $4 \mathrm{wt} . \%$ on glass substrates and drying under dynamic vacuum at $60^{\circ} \mathrm{C}$. The resulting films ( $6 \mu \mathrm{m}$ of thickness) were doped by dipping in 0 , $25 \mathrm{M}$ dimethylsulfoxide aqueous solution of sulfanilic acid for various period of time; $2 \mathrm{~h}, 6 \mathrm{~h}, 14 \mathrm{~h}, 24 \mathrm{~h}, 30 \mathrm{~h}$ and $72 \mathrm{~h}$. Thin films of various thicknesses were elaborated by modifying the concentration of PANI solution from $1 \mathrm{wt} . \%$ to $4 \mathrm{wt} . \%$ keeping the same volume deposited. The operating conditions are summarized in Table 1.

\section{Preparation of composite PANI/PVC films}

PVC and PANI were dissolved in NMP separately to prepare 20 wt.\% and $4 \mathrm{wt} . \%$ solutions, respectively. These fractions were selected in order to get solutions in form of gel to use spin coating technique. The Sulphanilic Acid (AS) was then added to the solution of PANI ${ }_{\mathrm{EB}}$, followed by 4 days stirring, with $[\mathrm{AS}] /\left[\mathrm{PANI}_{\mathrm{EB}}\right.$ ] ratio equals to 1 . This ratio is calculated by using a molar weight of PANI equals to $90,5 \mathrm{~g} /$ mol. Conducting polymer composites were prepared by mixing the above solutions with various weight percentages i.e. $2,5,8,11,14,17$ and $20 \mathrm{wt} . \%$ of PANI. The resulted well-mixed solutions were deposited on glass substrates using the spin coating technique, in static mode, at

*Corresponding author: Saad Lamouri, Laboratoire de Chimie Macromoléculaire, Ecole Militaire Polytechnique, BP 17, Bordj El Bahri, 16111 Algeria, Tel: 021-86-34-69; E-mail: lamourisaad@gmail.com

Received March 24, 2014; Accepted April 26, 2014; Published April 30, 2014

Citation: Lamouri S, Bendahgane S, Oudia A (2014) The Preparation and Analytical Study of Conducting Polyaniline Thin Films. J Pet Environ Biotechnol 5: 171. doi:10.4172/2157-7463.1000171

Copyright: ( $) 2014$ Lamouri S, et al. This is an open-access article distributed under the terms of the Creative Commons Attribution License, which permits unrestricted use, distribution, and reproduction in any medium, provided the original author and source are credited. 
Citation: Lamouri S, Bendahgane S, Oudia A (2014) The Preparation and Analytical Study of Conducting Polyaniline Thin Films. J Pet Environ Biotechnol 5: 171. doi:10.4172/2157-7463.1000171

a speed of $1000 \mathrm{rpm}$. Finally, the obtained films were dried at $\sim 60^{\circ} \mathrm{C}$. The film thickness was controlled by changing the rotation time of the spin-coater.

\section{Measurements}

Conductivity of the resulting films was determined by four-point probe method, by using a Keithley current source (Model 220) and a Keithley voltmeter (Model 619). Conductivity was calculated by the following equation [9]:

$$
\sigma=\frac{\ln 2}{\pi e} \times \frac{1}{V}
$$

Where $\sigma$ is the conductivity $(\mathrm{S} / \mathrm{cm}) ; \mathbf{V}$ is the potential difference $(\mathrm{V})$; $\mathbf{I}$ is the applied current $(\mathrm{A})$ and $\mathbf{e}$ is the thickness of the film $(\mathrm{cm})$.

Electrical conductivity and photoconductivity in the temperature range of $298-393 \mathrm{~K}$ (at heating rate $4^{\circ} \mathrm{C} / \mathrm{min}$ ) were measured by using a Keithley electrometer (Model 617) which measures the current across the films by applying voltage of $7 \mathrm{~V}$. Metal contacts were deposited by

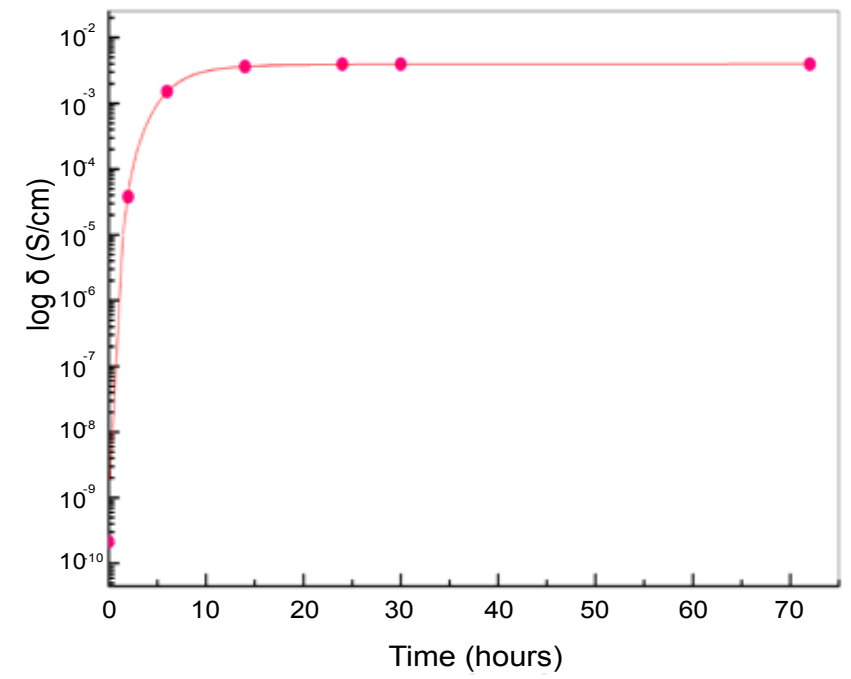

Figure 1: Relationship between the electrical conductivity and doping time.

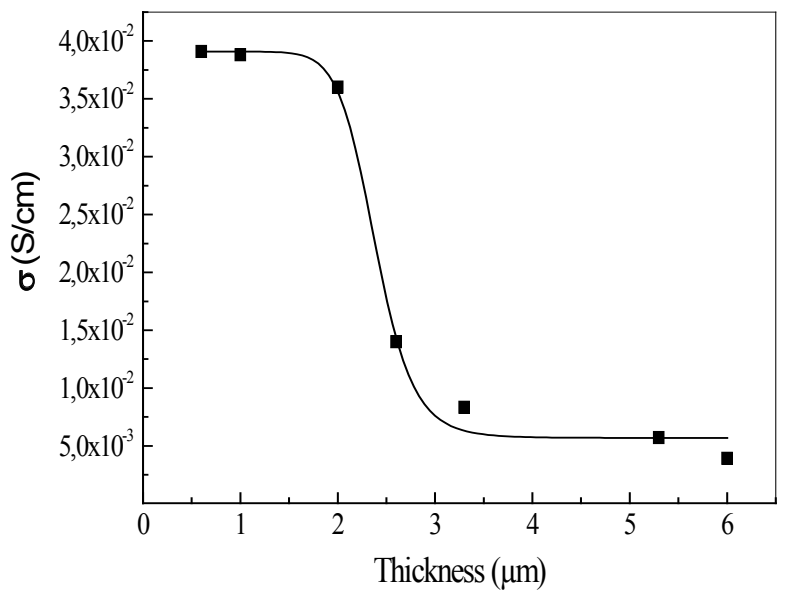

Figure 2: Evolution of conductivity as a function of film thickness.

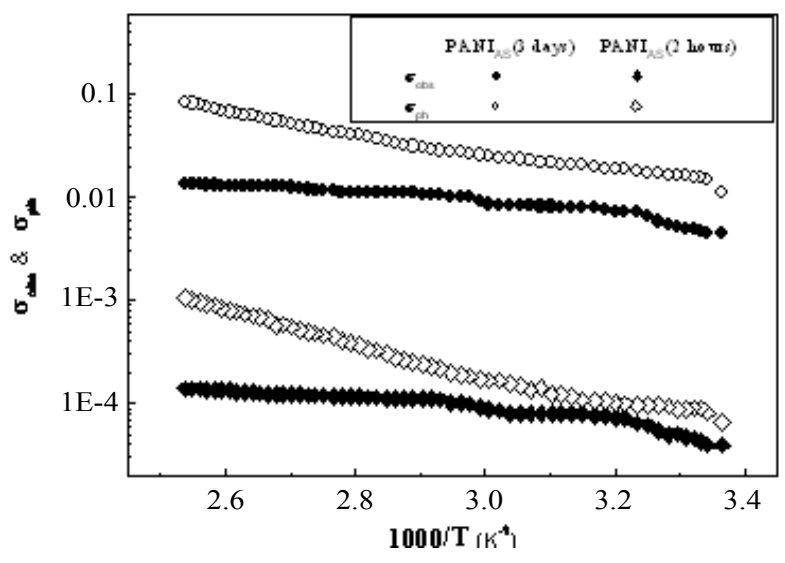

Figure 3: Variation of $\sigma_{\text {dark }}$ and $\sigma_{\text {ph }}$ vs 1000/T for PANIAS doped for 2 hours and PANIAS doped for 3 days.

evaporation. Fourier Transform Infrared (FTIR) spectra were obtained by using a SHIMADZU $8400 \mathrm{~S}$ in the range of $4000-400 \mathrm{~cm}^{-1}$ at a resolution of $1 \mathrm{~cm}^{-1}$ and 64 scans. A SETARAM TGA was used to investigate the thermal stability of films in the temperature range from 25 to $500^{\circ} \mathrm{C}$ under air atmosphere at a programmed heating rate of $10 \%$ min. The DSC spectra was measured with a NETZSCH DSC 204 $\mathrm{F} 1$ under a nitrogen purge at $10^{\circ} \mathrm{C} / \mathrm{min}$. Information about surface morphology was obtained by using a Quanta 600 scanning electron microscope with high voltage of $15 \mathrm{KV}$.

\section{Results and Discussion}

\section{Characterization of polyaniline films}

Figure 1 shows the electrical conductivity of films doped for many times of dipping. The conductivity increases according to doping time to stabilize after 24 hours of dipping at a value equal to $3.95 \times 10^{-3} \mathrm{~S} / \mathrm{cm}$. This value can increase up to $3.88 \times 10^{-2} \mathrm{~S} / \mathrm{cm}$ when the thickness value decreases from 3.3 to $1.6 \mu \mathrm{m}$ as shown in Figure 2 .

The Figure 3 presents the electrical conductivity measured in darkness $\sigma_{\text {dark }}$ as well as the photoconductivity $\sigma_{\text {ph }}$ (i.e. conductivity measured in presence of light) in the temperature range for two PANI films doped by dipping during 2 hours and 3 days, respectively. The plots of $\ln \sigma$ Vs 1000/T are straight lines; indicating that conduction in these samples is through an activated process having single activation energy in the temperature range $298-393 \mathrm{~K}$. The conductivity relation can be expressed by the following expression [10-12].

$$
\sigma_{\text {dark }}=\sigma_{0} e^{\left(-\mathrm{E}_{\mathrm{a}} / \mathrm{K}_{B}^{T}\right)}
$$

Where $\mathrm{k}_{\mathrm{B}}$ is the Boltzmann constant, $\sigma_{0}$ is the exponential factor and $\mathrm{E}_{\mathrm{a}}$ is the activation energy.

The results show that the PANI films are photoconductive because, for the two samples, the $\sigma_{\mathrm{ph}}$ curves were located on top of $\sigma_{\text {dark }}$ curves. However, the gap between these two conductivities depends on the doping time and temperature; it is more pronounced at high temperatures.

The FTIR characterization (Figure 4) was done for PANI films doped for various dipping times. IR spectra of undoped PANI reveals the existence of residual solvent (NMP) considering the appearance of a peak at around $1670 \mathrm{~cm}^{-1}$ assigned to the $(\mathrm{C}=\mathrm{O})$ stretching. The Occurrence of residual solvent is due to its high boiling point $\left(202^{\circ} \mathrm{C}\right)$ and the presence of hydrogen-bonding interaction between the $(\mathrm{C}=\mathrm{O})$ 


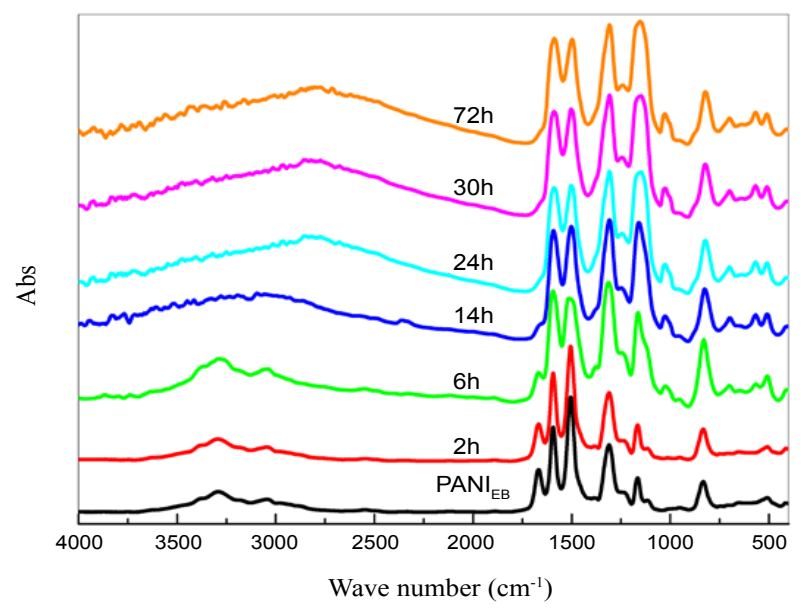

Figure 4: FTIR spectra of PANI films doped for various dipping times.

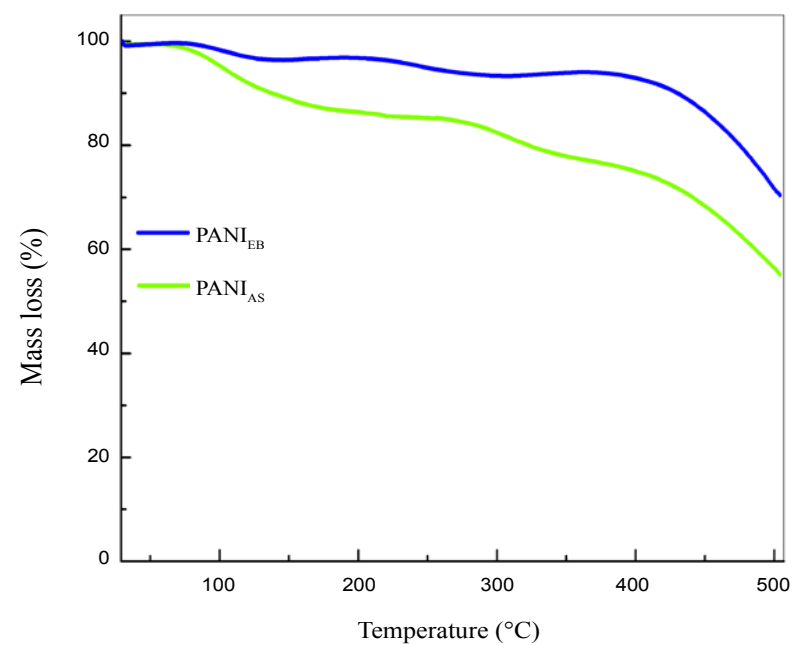

Figure 5: TGA curves of undoped and doped PANI.

group in NMP and $(\mathrm{NH})$ group in PANI [13]; the intensity of $(\mathrm{C}=\mathrm{O})$ stretching decreases with the increase in doping time and almost disappears after 24 hours of dipping, which corroborates the electrical conductivity results obtained previously. These results indicate the presence of a strong affinity between the doping acid and the NMP that prevents the interaction between the PANI and the NMP, which explains the disappearance of residual NMP after dipping for long times. The presence of two peaks located at 1027 and $691 \mathrm{~cm}^{-1}$ are attributed to the $(\mathrm{S}=\mathrm{O})$ and $(\mathrm{C}-\mathrm{S})$ vibration, respectively. These absorption bands confirm the protonation of the polyaniline.

Figure 5 presents the TGA measurements results of undoped and doped PANI. It shows that the thermal stability of the conducting form of PANI is less stable than the undoped one. According to the PANI ${ }_{\mathrm{EB}}$ thermogram, the residual amount of NMP is about $3 \%$ by weight.

\section{Characterization of composites PANI/PVC films}

The electrical conductivity of each film was measured and found to depend strongly on the fraction of polyaniline in the composite (Figure 6). The conductivity of the composite is lower than the conductivity of the doped PANI, which is due to the insulating nature of PVC. The composite conductivity was found to increase as the percentage of PANI increased in the composite. The increase in conductivity may be due to the increase of localized states in the band gap [10].

The addition of PANI in the matrix with a rate of $20 \mathrm{wt} . \%$ gives composite films $10^{3}$ times more conductive. The percolation threshold of composites is observed at around $16 \mathrm{wt} . \%$.

It is clearly shown in Figure 7 that the electrical conductivity can be 1.5 times greater when the film thickness value decreases from 3.3 to $1 \mu \mathrm{m}$. Also the conductivity of composites films increases with the increase of temperature. For the semiconductor sample containing 17 wt. $\%$ of PANI, there is linearity with the temperature which corresponds to an Arrhenius behavior [10]. Whereas, for the insulating sample containing $2 \mathrm{wt} . \%$ of PANI the temperature has effect only beyond $70^{\circ} \mathrm{C}$ (Figure 8).

Thus, these two composite films exposed to light have conductivities values higher than those measured in darkness, indicating that these samples are photoconductive.

The IR absorption spectra were used to determine the way of interaction between the two polymers in the composites [14]. Figure 9 presents the FTIR spectra of PVC and PANI/PVC composites. This figure shows that the characteristic bands of PANI appear on the composites spectra starting from a rate of $5 \mathrm{wt} . \%$ of PANI and become more intense with higher rates; quoting as an example the two bands

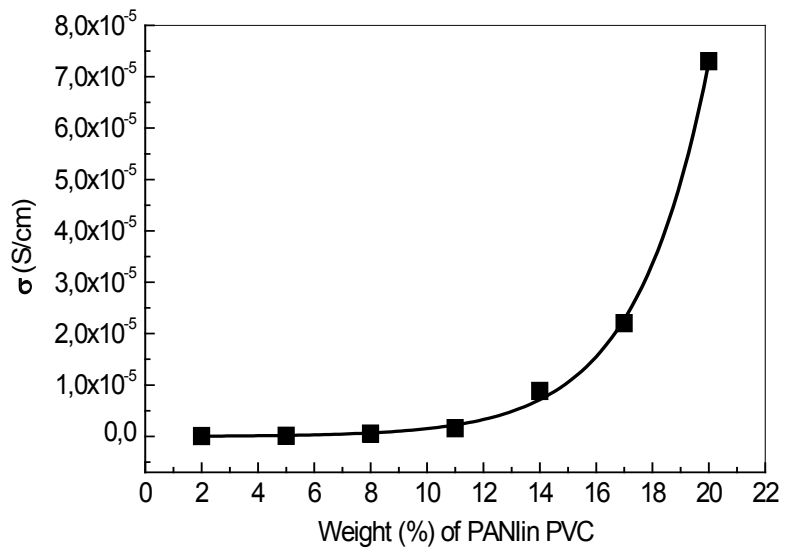

Figure 6: Conductivity vs. PANI content in the composite PANI-PVC

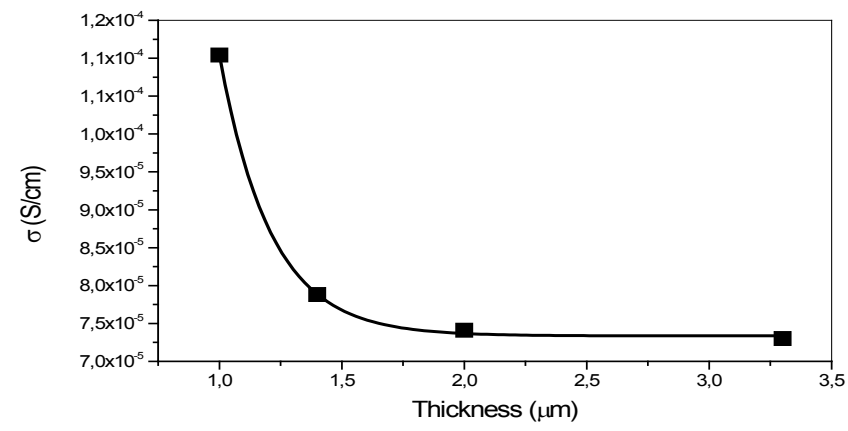

Figure 7: Relationship between the electrical conductivity and film thickness for composite content 20 wt. $\%$ of PANI. 
Citation: Lamouri S, Bendahgane S, Oudia A (2014) The Preparation and Analytical Study of Conducting Polyaniline Thin Films. J Pet Environ Biotechnol 5: 171. doi:10.4172/2157-7463.1000171

Page 4 of 5

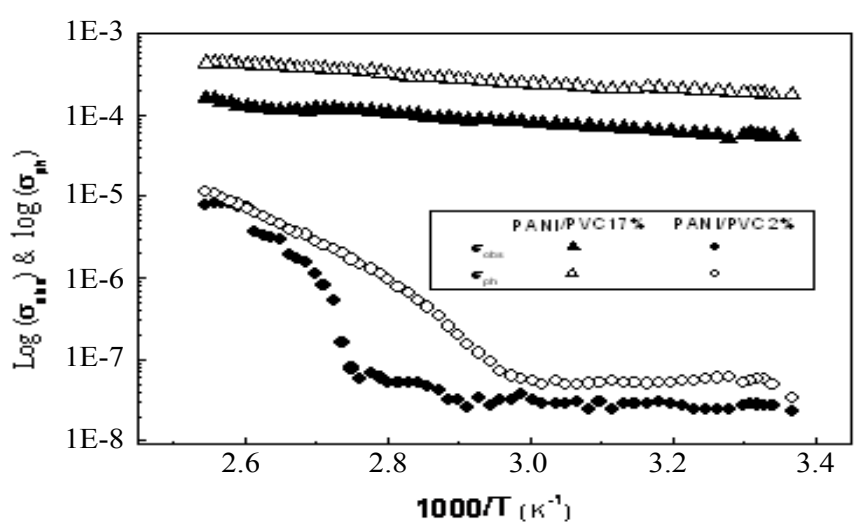

Figure 8: Variation of $\sigma_{\text {dark }}$ and $\sigma_{\text {ph }}$ vs $1000 / T$ for PANI-PVC composites containing 2 wt. $\%$ and 17 wt. $\%$ of PANI.

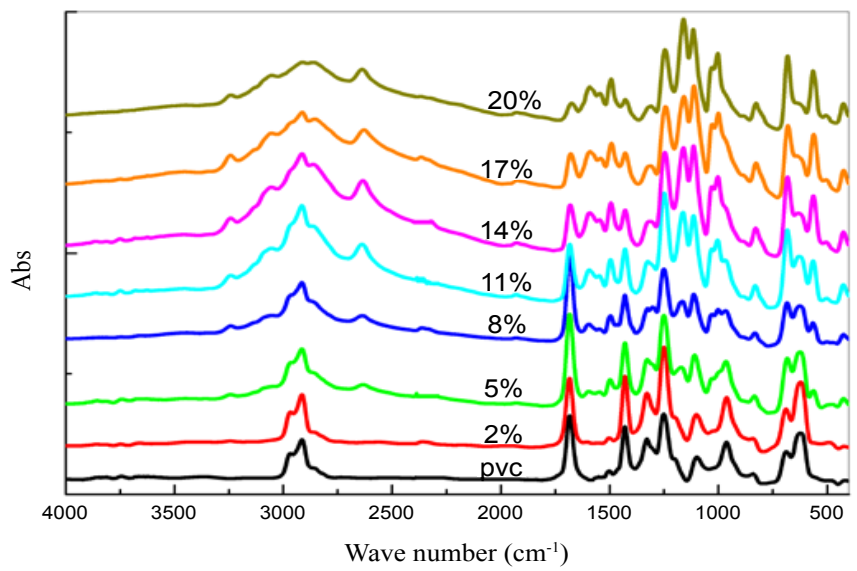

Figure 9: FTIR spectra of pure PVC and PANI/PVC composites with different weight fraction of PANI.

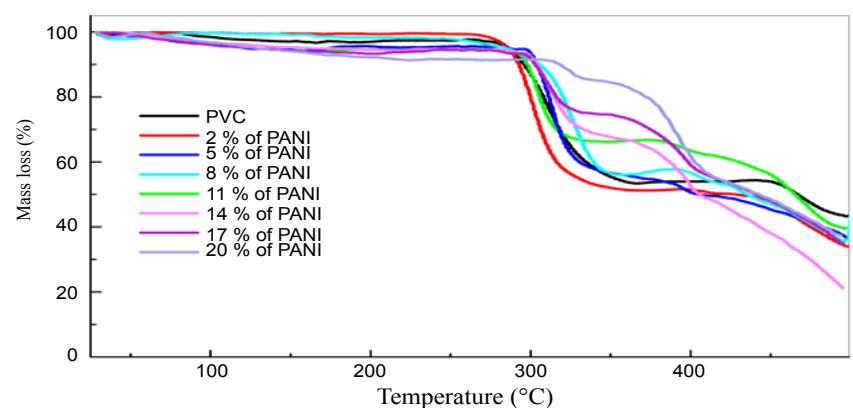

Figure 10: TGA curves of PANI/PVC composites.

located at about 1592 and $1489 \mathrm{~cm}^{-1}$, assigned to the stretching of quinoid and benzoid rings, respectively. The band observed at $685 \mathrm{~cm}^{-1}$ is assigned to the $(\mathrm{C}-\mathrm{Cl})$ stretching in pure PVC. The mixing of PANI in PVC causes this band to shift to lower wave numbers. The higher the PANI content in the composite, the lower is the wave num/ober. In composite with $20 \mathrm{wt} . \%$ of PANI, this band is shifted to $677 \mathrm{~cm}^{-1}$; i.e. $8 \mathrm{~cm}^{-1}$ lower than the pure PVC. Moreover, it reveals a peak at around $3055 \mathrm{~cm}^{-1}$, these two observations may be ascribed to the chemical interaction (strong dipole-dipole interaction) between $(\mathrm{C}-\mathrm{Cl})$ group of PVC and amine group of PANI. Similar results have been reported by Afzal et al. [15].

Thus, the $(\mathrm{C}=\mathrm{O})$ group revealed at $1681 \mathrm{~cm}^{-1}$ on PVC spectra confirm the existence of residual solvent (NMP) in result films. The mixing of PANI in PVC causes this band intensity to decrease; this is probably due to the interactions between the two polymers which gives a steric obstruction preventing the interaction between PVC and NMP.

Results of TGA measurements of the pure PVC and PANI/PVC composites are shown in Figure 10. The PVC film thermogram presents three-step mass loss process. The first one is due to the evaporation of residual NMP (result confirmed by IRTF). While the second (more important) is attributed to the dehydrochlorination process, followed by several simultaneous polyene rearrangement reactions [16-18]. Whereas the composites thermograms show four- step mass loss process. The first loss is attributed to the evaporation of water and residual NMP. The second step corresponds to the loss of $\mathrm{HCl}$ released from the dehydrochlorination of PVC. This loss decreases with the addition of PANI which prove that the mixing of PANI in PVC improve the thermal stability. The third process is due to the degradation of doping agent. Finally the last step corresponds to the beginning of degradation of the two polymers chains. DSC results of the pure PVC film and PANI/PVC composite films are shown in Figure 11.

Table 2 shows the glass transition values of various composites PANI/PVC.

The variation in the glass transition of PANI/PVC composites according to the PANI fraction shows a positive deviation compared to the glass transition of PVC. This deviation represents the increase in the rigidity of composites by the addition of PANI as well as the existence of interactions between the two polymers.

Figure 12 represents the SEM images of PANI/PVC composites at fractions of 5, 11 and 20 wt.\% of PANI. The films structure observed

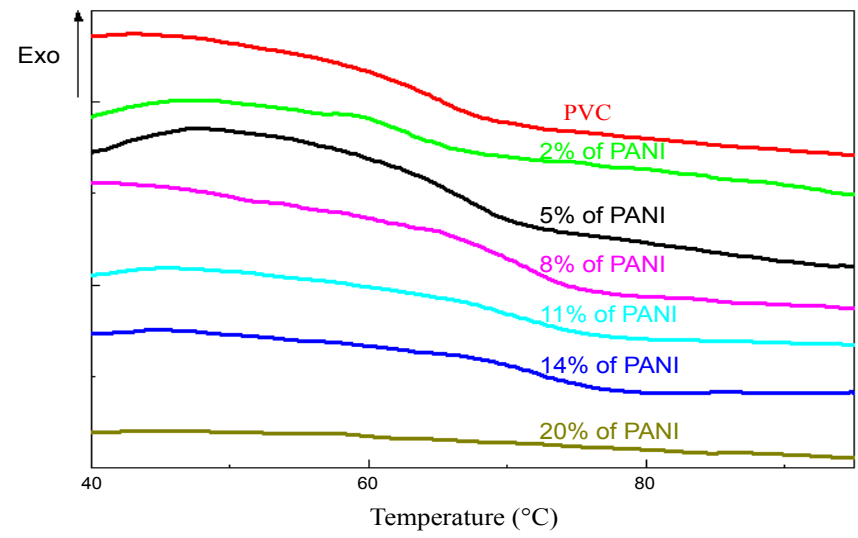

Figure 11: The DSC thermograms of pure PVC and PANI/PVC composites.

\begin{tabular}{|c|c|}
\hline Composite films PANI/PVC & Glass transition Tg $\left({ }^{\circ} \mathbf{C}\right)$ \\
\hline 2 wt. $\%$ of PANI & 2 wt. $\%$ of PANI \\
\hline 5 wt. $\%$ of PANI & 5 wt. $\%$ of PANI \\
\hline 8 wt. $\%$ of PANI & 8 wt. $\%$ of PANI \\
\hline 11 wt. $\%$ of PANI & 11 wt. $\%$ of PANI \\
\hline 14 wt. $\%$ of PANI & 14 wt. $\%$ of PANI \\
\hline $17 w t . \%$ of PANI & 17 wt. $\%$ of PANI \\
\hline 20 wt. $\%$ of PANI & 20 wt. $\%$ of PANI \\
\hline
\end{tabular}

Table 2: Fraction PANI effect on the glass transition of composites films. 

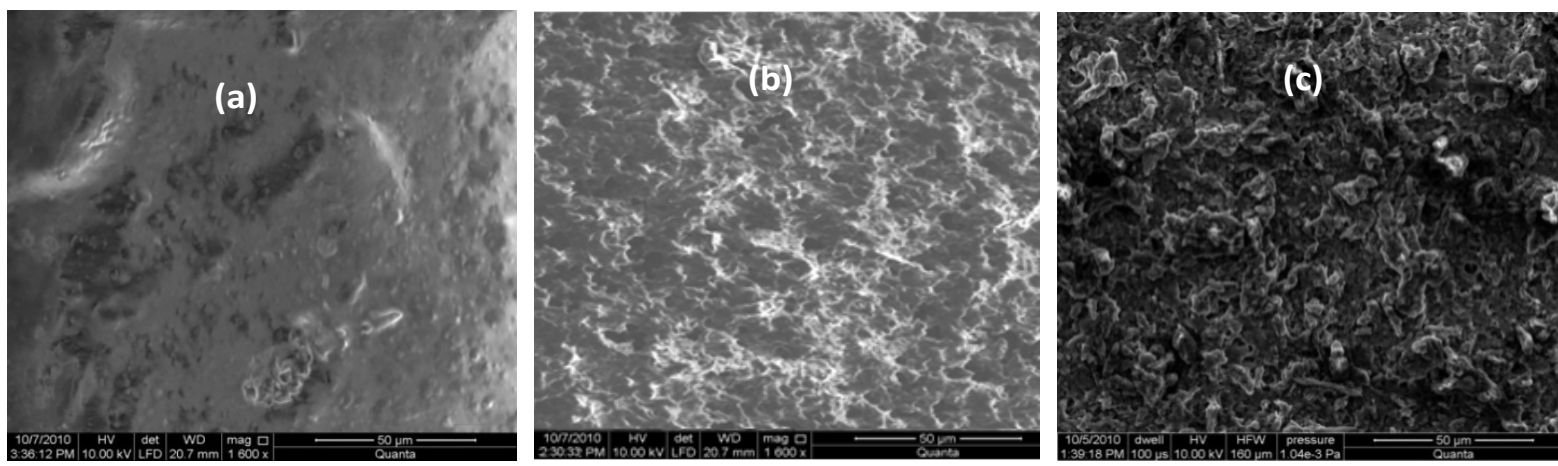

Figure 12: SEM images of PANI/PVC composites. (a). $5 \%$ of PANI, (b). $11 \%$ of PANI, (c). $20 \%$ of PANI.

by SEM shows a clear evolution of morphology according to the PANI fraction. Indeed, the roughness of surface appears for high fractions of PANI.

\section{Conclusion}

The electrical conductivity of PANI films increased according to the dipping time in sulfanilic acid solution and stabilized after 24 hours of dipping at a value equals to $3,95 \cdot 10^{-3} \mathrm{~S} / \mathrm{cm}$. This value increased significantly with the decrease of film thickness. The characteristic conductivity-temperature indicated the photoconductive behavior of PANI films doped with sulphanilic acid. The examination of FTIR spectra and TGA curves showed the presence of residual solvent in $\mathrm{PANI}_{\mathrm{FB}}$ films. The residual NMP could disappear after doping by dipping in sulfanilic acid solution for 24 hours. PANI/PVC composite films with a good flexibility were prepared in NMP using solution blending method. The obtained films had a percolation threshold of 16 wt.\% of PANI. FTIR spectroscopy reveals that PVC and PANI strongly interact with each other in the composites through hydrogen bond interactions. The mixing of PANI in PVC causes the increase of thermal stability as well as rigidity of composite PANI/PVC.

\section{References}

1. Djurado D, Bée M, Gonzalez M, Mondelli C, Dufour B, et al. (2003) Molecular dynamics in plastic conducting compounds of polyaniline. Chem Phys 292: 355-361

2. Namazi H, Kabiri R, Entezami A (2002) Determination of extremely low percolation threshold electroactivity of the blend polyvinyl chloride/polyaniline doped with camphorsulfonic acid by cyclic voltammetry method. Eur Polym J 38: 771-777.

3. Olinga T, Pron A, Travers JP (2002) Use of sulphonic and phosphonic acids as dopants of conductive polyaniline films and conductive composite materials based on polyaniline. EP $1194932 \mathrm{~A} 1$.

4. Han MG, Lee YJ, Byun SW, Soon Im S (2001) Physical properties and thermal transition of polyaniline film. Synthetic Met 124: 337-343.

5. Ayad MM, Zaki EA (2008) Doping of polyaniline films with organic sulfonic acids in aqueous media and the effect of water on these doped films. Eur Polym $J$ 44: $3741-3747$

6. Sniechowski M, Djurado D, Dufour B, Rannou P, Pron A, et al. (2004) Direct analysis of lamellar structure in polyaniline protonated with plasticizing dopants. Synthetic Met 143: 163-169.

7. Paul RK, Pillai CKS (2000) Melt/solution processable conducting polyaniline with novel sulfonic acid dopants and its thermoplastic blends. Synthetic Met 114: 27-35.
8. Jayashree A, Srinivasan P, Sathyanarayana DN (1998) Conducting polyaniline blends and composites. Prog Polym Sci 23: 993-1018.

9. Heaney MB (2000) Electrical Conductivity and Resistivity. CRC Press LLC.

10. Ameen S, Ali V, Zulfequar M, Mazharul Haq M, Husain M (2008) Electrical and spectroscopic characterization of polyaniline-polyvinyl chloride (PANI-PVC) blends doped with sodium thiosulphate. Physica B: Condensed Matter 403 2861-2866.

11. Afzal AB, Akhtar MJ, Svensson LG (2010) Thermal studies of DBSA-doped polyaniline/PVC blends by isothermal microcalorimetry. J Therm Anal Calorim 100: 1017-1025.

12. Farrokhzad H, Van Gerven T, Van der Bruggen B (2013) Preparation and characterization of a conductive polyaniline/polysulfone film and evaluation of the effect of co-solvent. Eur Polym J 49: 3234-3243.

13. Chen SA, Lee HT (1993) Polyaniline plasticized with 1-methyl-2-pyrrolidone: structure and doping behavior. Macromolecules 26: 3254-3261.

14. Singh V, Mohan S, Singh G, Pandey PC, Prakash R (2008) Synthesis and characterization of polyaniline-carboxylated PVC composites: Application in development of ammonia sensor. Sensor Actuat B-Chem 132: 99-106.

15. Afzal AB, Akhtar MJ, Nadeem M, Hassan MM (2010) Dielectric and impedance studies of DBSA doped polyaniline/PVC composites. Current Applied Physics 10: $601-606$

16. Magnin G, Sergent M, Coen S, Périchaud A, Phan-Tan-Luu R (2000) Degradation Thermique des Polystyrenes et du Polychlorure de Vinyle Application d'une Matrice d'experiences a l'etude de l'influence des Parametres de l'analyse Thermomanometrique. Partie I : Exploitation des résultats par traitement mathématique. J Therm Anal Calorim 61: 51-61.

17. Pielichowski K (1998) Thermal Degradation of Poly(Vinyl Chloride)/Polyaniline Conducting Blends. J Therm Anal Calorim 54: 171-175.

18. Afzal AB, Akhtar MJ (2012) Effects of silver nanoparticles on thermal properties of DBSA-doped polyaniline/PVC blends. Iran Polym J 21: 489-496. 